\title{
Adoption of Good Agriculture Practices by Mango Growers
}

\author{
Y. J. Waghmod ${ }^{1 *}$, D. P. Hardikar ${ }^{2}$ and P. C. Haldavanekar ${ }^{3}$ \\ Department of Extension Education' Dr. Balasaheb Sawant Konkan Krishi Vidyapeeth, \\ Dapoli, Dist: Ratnagiri State: Maharashtra, India \\ *Corresponding author
}

\section{A B S T R A C T}

\begin{tabular}{l} 
Ke y w o r d s \\
$\begin{array}{l}\text { Global Gap, Mango } \\
\text { growers, Attitude, } \\
\text { Konkan Region and } \\
\text { Certification etc. }\end{array}$ \\
\hline Article Info \\
\hline $\begin{array}{l}\text { Accepted: } \\
\text { 04 March } 2020 \\
\text { Available Online: } \\
\text { 10 April } 2020\end{array}$ \\
\hline
\end{tabular}

\section{Introduction}

In this scenario, the importance of microbial contamination is of major and has been the driving force behind the establishment of the USA Good Agricultural Practices (GAP) policies and surveillance systems. Currently, there are numerous systems that growers can adopt to ensure safe food production, which include amongst others Good Agricultural Practices (GAP), Good Manufacturing Practices (GMP), Hazard Analysis Critical Control Points (HACCP), Good Hygiene Practices, etc.

One of the GAP systems that have taken off within the European community is GLOBAL
GAP. The challenge of globalizing markets is nowhere greater than in the primary food sector. GLOBAL GAP (formerly known as EUREP GAP) has established itself as a key reference for Good Agricultural Practice (GAP) in the global market place, by translating consumer requirements into agricultural production in a rapidly growing list of countries currently more than 100 in each continent.

Apart from Germany and France, most other countries within the EU support this system, as do the major retailers, which consider it the minimum standard for food trade. It is important to note that these global standards will hopefully be harmonized but for the time 
being, major retailers will still have their own set of requirements that growers will have to adhere to.

The concept of Good Agricultural Practices may serve as a reference tool for deciding, at each step in the production process, on practices and/or outcomes that are environmentally sustainable and socially acceptable. A multiplicity of Good Agricultural Practices (GAP) codes, standards and regulations have been developed in recent years by the food industry and producers organizations, but also governments and NGOs, aiming to codify agricultural practices at farm level for a range of commodities. Their purpose varies from fulfillment of trade and government regulatory requirements (in particular with regard to food safety and quality), to more specific requirements of specialty or niche markets. The objective of these GAP codes, standards and regulations to a varying degree includes, ensuring safety and quality of produce in the food chain, capturing new market advantages by modifying supply chain governance, improving natural resources use, workers health and working conditions, and/or creating new market opportunities for farmers and exporters in developing countries. Good Agricultural Practices are 'practices that address environmental, economic and social sustainability for on-farm processes, and result in safe and quality food and non-food agricultural products' (FAO COAG 2003 GAP paper).

These four 'pillars' of GAP (economic viability, environmental sustainability, social acceptability and food safety and quality) are included in most private and public sector standards, but the scope which they actually cover varies widely. The implementation of GAP should therefore contribute to Sustainable Agriculture and Rural Development (SARD).
Good Agricultural Practices are a collection of principles to apply for on-farm production and post-production processes, resulting in safe and healthy food and non-food agricultural products, while taking into account economic, social and environmental sustainability. GAP may be applied to a wide range of farming systems and at different scales.

They are applied through sustainable agricultural methods, such as integrated pest management, integrated fertilizer management and conservation agriculture. They rely on four principles:

Economically and efficiently produce sufficient (food security), safe (food safety) and nutritious food (food quality).

Sustain and enhance natural resources.

Maintain viable farming enterprises and contribute to sustainable livelihoods.

Meet cultural and social demands of society.

\section{Adoption of good agriculture practices by mango growers}

An enquiry was made with the respondents about their willingness to adopt the good agriculture practices of mango. Based on their total score, they were grouped into three categories of symbolic adoption as shown in Table 1.

It is observed form Table 1 that nearly twothird (64.00 per cent) of the respondents had 'medium' adoption, while 19.00 per cent of the respondents had 'low' level of adoption and 17.00 per cent of the respondents had 'high' level of adoption. The mean adoption score was 70.29.

It can be concluded from these findings that majority of the respondents adopted the good agriculture practices. This may be probably because the respondents might be convinced about the utility of GAP 
Practice wise adoption of good agriculture practices by the mango growers

The responses of the respondents about their willingness to adopt practice wise good agriculture practices of mango are summarised in Table 2 (a) to 2 (j).

Good agricultural practices of farm location

The data with regard to the adoption of good agriculture practices of farm location by the mango growers are presented in Table 2 (a).

It is noticed from Table 2 (a) that majority of the respondents had adopted "prior land use of the farm evaluated for possible sources of chemical, biological or physical contaminants', 'soil analysed for possible heavy metal contamination', 'water analysis or water testing' practices 'completely' and near to cent per cent of the respondents adopted 'completely' practices like 'records of heavy metal analyses from government accredited / recognized laboratories must be kept and made available', while about soil and climatic conditions, cent per cent of the respondents adopted practices like $\mathrm{pH}$ of 5.5 6.5 , flat to slightly rolling terrain, distinct wet and dry climate, temperature between 16$34^{\circ} \mathrm{C}$ and elevation between 0 to $600 \mathrm{~m}$ from sea level.

\section{Good agriculture practices of farm structure and maintenance}

The data with regard to the adoption of good agriculture practices of farm structure and maintenance by the mango growers are presented in Table 2 (b).

It is observed from Table 2 (b) that cent per cent (100.00) of the respondents had 'complete' adoption about 'storage and packing areas are kept clean and tidy', 'effective measures are taken to dispense of the trash or waste material on the farm', 'all equipments are associated with cultivation, harvesting and storage of mangoes clean and maintained at optimum operating conditions'. While nearly cent per cent of the respondents had 'complete' adoption about 'farm boundaries are enclosed and demarcated by a fence and the entrance and exit to the farm have gates' and 'adequate areas for waste collection and storage for biodegradable and non-biodegradable wastes are provided'.

\section{Good agriculture practices of cultural management of small plants}

The data with regard to the adoption of good agriculture practices of cultural management of small plants by the mango growers are presented in Table 2 (c).

It is observed from Table 2 (c) that about quality planting material cent per cent $(100.00$ per cent) of the respondents had 'complete' adoption about 'chose planting materials based on resistance to pest, suitability to the site, yield potential and market preference', 'minimum age of the planting material preferably be one year old or at least $1 \mathrm{~m}$ high' and 'selection of variety', whereas 91.00 per cent of the respondents had 'complete' adoption about that 'record of the source of the planting materials, the number and date of purchase kept in the farm'.

\section{Good agriculture practices of cultural management of young trees}

The data with regard to the adoption of good agriculture practices of cultural management of young trees by the mango growers are presented in Table 2 (d).

It is observed from Table 2 (d) that with regards to early pruning about more than half of the respondents 'not adopted' practices like 'tree is about 1 to $2 \mathrm{~m}$ height, the terminal 
portions are cut/pinched to encourage lateral branching', 'three to four branches are allowed to grow to $1 \mathrm{~m}$ height', 'second cutting or pruning of terminal portions is done until the branches are evenly distributed', Whereas more than four-fifth (87.00 per cent) of the respondents had 'complete' adoption of 'removal of dead, diseased, infested and damaged branches'.

\section{Good agriculture practices of cultural management of bearing trees}

The data with regard to the adoption of good agriculture practices of cultural management of bearing trees by the mango growers are presented in Table 2 (e).

It is noticed from Table 2 (e) that with regards to pruning, it is observed that nearly threefourth (70.00 per cent) of the respondents had adopted 'completely' 'removal of unnecessary branches such as water sprouts, dried, disease-infected branches and all branches that grow crowding the canopy'. It is observed that more than two-third (70.00 per cent) of the respondents had 'no' adoption of 'pruning done during summer months or dry and warm days'.

\section{Good agriculture practices of integrated pest management}

The data with regard to the adoption of good agriculture practices of integrated pest management by the mango growers are presented in Table 2 (f).

It is observed from Table 2 (f) that for ants control cent per cent (100.00 per cent) of the respondents had 'complete' adoption of 'apply insecticides during heavy infestation', while 69.00 per cent of the respondents did not adopt 'prune the mango tree and remove all unnecessary branches that provide favourable environment for ants'.

With regards to management of fruit fly, it is noticed that majority of the respondents had 'complete' adoption of 'use of 'Rakshak' trap for controlling fruit fly' and 'collect the infested fruits and burry deep into the soil to prevent the insect from completing its life cycle', while 42.00 per cent of the respondents had 'complete' adoption of 'harvest fruits at mature green stage since fruit flies are attracted to them as soon as their surfaces become yellow'. Whereas in case of 'bag the fruits with appropriate bagging materials', 92.00 per cent of the respondents had 'no' adoption of this practice.

\section{Good agriculture practices of integrated disease management}

The data with regard to the adoption of good agriculture practices of integrated disease management by the mango growers are presented in Table $2(\mathrm{~g})$.

It is observed from Table $2(\mathrm{~g})$ that in case anthracnose disease, all (100.00 per cent) of the respondents had 'complete' adoption of 'spray fungicides for control of anthracnose' and 91.00 per cent of the respondents had 'complete' adoption of 'use spray schedule'.

With regards to powdery mildew, it is observed that more than four-fifth $(94.00$ per cent) of the respondents had 'complete' adoption of 'use spray schedule'. Whereas, more than four-fifth ( 83.00 per cent) of the respondents had 'no' adoption of 'use of sulphur smoke'. 
Table.1 Distribution of the respondents according to their overall adoption

\begin{tabular}{|l|l|c|c|}
\hline \multirow{2}{*}{ Sl.No. } & \multicolumn{1}{|c|}{ Adoption (score) } & \multicolumn{2}{|c|}{ Respondents (N= 100) } \\
\cline { 3 - 4 } & & Number & Percentage \\
\hline 1. & Low (up to 64) & 19 & 19.00 \\
\hline 2. & Medium (65 to 75) & 64 & 64.00 \\
\hline 3. & High (76 and above) & 17 & 17.00 \\
\hline \multicolumn{2}{|r|}{ Mean- 70.29 } & $\mathbf{1 0 0}$ & $\mathbf{1 0 0 . 0 0}$ \\
\hline
\end{tabular}

Table.2 (a) Distribution of the respondents according to adoption of good agriculture practices of farm location

\begin{tabular}{|c|c|c|c|c|}
\hline \multirow[t]{2}{*}{ Sl. No. } & \multirow[t]{2}{*}{ Practices } & \multicolumn{3}{|c|}{ Adoption $(\mathrm{N}=100)$} \\
\hline & & Complete & Partial & No \\
\hline 1 & $\begin{array}{l}\text { Prior land use of the farm evaluated for possible } \\
\text { sources of chemical, biological or physical } \\
\text { contaminants }\end{array}$ & $\begin{array}{c}94 \\
(94.00)\end{array}$ & $06(6.00)$ & -- \\
\hline 2 & $\begin{array}{l}\text { Soil analysed for possible heavy metal } \\
\text { contamination }\end{array}$ & $\begin{array}{c}87 \\
(87.00)\end{array}$ & $\begin{array}{c}13 \\
(13.00)\end{array}$ & -- \\
\hline 3 & Water analysis or Water testing & $\begin{array}{c}86 \\
(86.00)\end{array}$ & $\begin{array}{c}14 \\
(14.00)\end{array}$ & -- \\
\hline \multirow[t]{10}{*}{4} & $\begin{array}{l}\text { Records of heavy metal analyses from } \\
\text { government accredited / recognized laboratories } \\
\text { must be kept and made available }\end{array}$ & $\begin{array}{c}95 \\
(95.00)\end{array}$ & $\begin{array}{c}05 \\
(5.00)\end{array}$ & -- \\
\hline & \multicolumn{4}{|l|}{ a. Soil characteristics } \\
\hline & 1. $\mathrm{pH}$ of $5.5-6.5$ & $\begin{array}{c}100 \\
(100.00)\end{array}$ & -- & -- \\
\hline & 2. Flat to slightly rolling terrain & $\begin{array}{c}64 \\
(64.00)\end{array}$ & $\begin{array}{c}36 \\
(36.00)\end{array}$ & -- \\
\hline & \multicolumn{4}{|l|}{ b. Climatic conditions } \\
\hline & 1. Distinct wet and dry climate & $100(100.00)$ & -- & -- \\
\hline & 2. Temperature between $16-34^{\circ} \mathrm{C}$ & $100(100.00)$ & -- & -- \\
\hline & 3. Avoid plant from strong winds and typhoons & $\begin{array}{c}44 \\
(44.00)\end{array}$ & $05(5.00)$ & $\begin{array}{c}51 \\
(51.00)\end{array}$ \\
\hline & \multicolumn{4}{|l|}{ c. Elevation } \\
\hline & Elevation between 0 to $600 \mathrm{~m}$ from sea level & $100(100.00)$ & -- & -- \\
\hline 5 & $\begin{array}{l}\text { Farm topography map made available showing } \\
\text { the location of crop production sites, } \\
\text { windbreakers, water resources, irrigation lines, } \\
\text { drainage canals and outlets, roads, buildings, } \\
\text { storage facilities and other farm structures }\end{array}$ & $\begin{array}{c}98 \\
(98.00)\end{array}$ & $02(2.00)$ & -- \\
\hline
\end{tabular}


Table.2 (b) Distribution of the respondents according to adoption of good agriculture practices of farm structure and maintenance

\begin{tabular}{|c|c|c|c|c|}
\hline \multirow[t]{2}{*}{ Sl. No. } & \multirow[t]{2}{*}{ Practices } & \multicolumn{3}{|c|}{ Adoption $(\mathrm{N}=100)$} \\
\hline & & Complete & Partial & No \\
\hline 1 & $\begin{array}{l}\text { Farm boundaries are enclosed and demarcated by a } \\
\text { fence and the entrance and exit to the farm have gates }\end{array}$ & $\begin{array}{c}98 \\
(98.00)\end{array}$ & $\begin{array}{c}02 \\
(2.00)\end{array}$ & -- \\
\hline 2 & $\begin{array}{l}\text { Designated areas or facilities for the working shed of } \\
\text { workers and separate sheds for farm implements and } \\
\text { equipments are available }\end{array}$ & $\begin{array}{c}94 \\
(94.00)\end{array}$ & $\begin{array}{c}03 \\
(3.00)\end{array}$ & $\begin{array}{c}03 \\
(3.00)\end{array}$ \\
\hline 3 & $\begin{array}{l}\text { Toilet is provide for the farm workers with proper } \\
\text { maintenance }\end{array}$ & $\begin{array}{r}92 \\
(92.00)\end{array}$ & $\begin{array}{c}08 \\
(8.00)\end{array}$ & -- \\
\hline 4 & Storage and packing areas are kept clean and tidy & $\begin{array}{c}100 \\
(100.00)\end{array}$ & -- & -- \\
\hline 5 & $\begin{array}{l}\text { Litter and waste materials are removed immediately } \\
\text { from the crop production area }\end{array}$ & $\begin{array}{c}65 \\
(65.00)\end{array}$ & $\begin{array}{c}35 \\
(35.00)\end{array}$ & -- \\
\hline 6 & $\begin{array}{l}\text { Effective measures are taken to dispense of the trash or } \\
\text { waste material on the farm }\end{array}$ & $\begin{array}{c}100 \\
(100.00)\end{array}$ & -- & -- \\
\hline 7 & $\begin{array}{l}\text { Irrigation system clean and well-maintained to provide } \\
\text { effective delivery of clean water, prevent blockage and } \\
\text { backflow }\end{array}$ & $\begin{array}{c}73 \\
(73.00)\end{array}$ & $\begin{array}{c}27 \\
(27.00)\end{array}$ & -- \\
\hline 8 & $\begin{array}{l}\text { All equipments are associated with cultivation, } \\
\text { harvesting and storage of mangoes are clean and } \\
\text { maintained at optimum operating conditions }\end{array}$ & $\begin{array}{c}100 \\
(100.00)\end{array}$ & -- & -- \\
\hline 9 & $\begin{array}{l}\text { Adequate areas for waste collection and storage for } \\
\text { biodegradable and non-biodegradable wastes are } \\
\text { provided }\end{array}$ & $\begin{array}{c}95 \\
(95.00)\end{array}$ & $\begin{array}{c}05 \\
(5.00)\end{array}$ & -- \\
\hline 10 & $\begin{array}{l}\text { Domestic and farm animals are excluded from the } \\
\text { production site and packing shed during harvesting } \\
\text { season }\end{array}$ & $\begin{array}{c}75 \\
(75.00)\end{array}$ & $\begin{array}{c}25 \\
(25.00)\end{array}$ & -- \\
\hline
\end{tabular}


Table.2 (c) Distribution of the respondents according to adoption of good agriculture practices of cultural management of small plants

\begin{tabular}{|c|c|c|c|c|}
\hline \multirow{2}{*}{$\begin{array}{l}\text { Sl. } \\
\text { No. }\end{array}$} & \multirow{2}{*}{ Practices } & \multicolumn{3}{|c|}{ Adoption $(\mathrm{N}=100)$} \\
\hline & & Complete & Partial & No \\
\hline A. & \multicolumn{4}{|l|}{ Quality planting materials } \\
\hline 1 & $\begin{array}{l}\text { Choose planting materials based on resistance to } \\
\text { pest, suitability to the site, yield potential and } \\
\text { market preference }\end{array}$ & $100(100.00)$ & -- & -- \\
\hline 2 & $\begin{array}{l}\text { Record of the source of the planting materials, the } \\
\text { number and date of purchase kept in the farm }\end{array}$ & $91(91.00)$ & $9(9.00)$ & -- \\
\hline 3 & $\begin{array}{l}\text { Minimum age of the planting material preferably } \\
\text { be one year old or at least } 1 \mathrm{~m} \text { high }\end{array}$ & $100(100.00)$ & -- & -- \\
\hline \multirow[t]{6}{*}{4} & Selection of variety & & & \\
\hline & i. Alphonso & $100(100.00)$ & -- & -- \\
\hline & ii. Kesar & -- & $\begin{array}{c}28 \\
(28.00)\end{array}$ & -- \\
\hline & iii. Ratna & -- & $\begin{array}{c}68 \\
(68.00)\end{array}$ & -- \\
\hline & iv. Sindhu & -- & $\begin{array}{c}18 \\
(18.00)\end{array}$ & -- \\
\hline & $\begin{array}{l}\text { v. Other- 1. Payri 2. Totapuri } \\
\text { 3. Langada }\end{array}$ & -- & $\begin{array}{c}28 \\
(28.00)\end{array}$ & -- \\
\hline B. & \multicolumn{4}{|l|}{ Land preparation } \\
\hline 1 & $\begin{array}{l}\text { For steeper areas, practice minimum tillage } \\
\text { around planting place by clearing about } 2 \mathrm{sq} . \mathrm{m}\end{array}$ & $91(91.00)$ & $9(9.00)$ & -- \\
\hline 2 & $\begin{array}{l}\text { Land preparation is best done before the onset of } \\
\text { the rainy season }\end{array}$ & $78(78.00)$ & $\begin{array}{c}17 \\
(17.00)\end{array}$ & $5(5.00)$ \\
\hline 3 & For each plant a pit is dig at least $1 \times 1 \times 1 \mathrm{cu} . \mathrm{m}$. & $82(82.00)$ & $\begin{array}{c}18 \\
(18.00)\end{array}$ & -- \\
\hline 4 & $\begin{array}{l}\text { Refill the pit with rich top soil and fully } \\
\text { decomposed organic compost at a ratio of } 3 \text { parts } \\
\text { top soil and } 1 \text { part organic compost with minerals }\end{array}$ & $89(89.00)$ & $\begin{array}{c}11 \\
(11.00)\end{array}$ & -- \\
\hline C. & \multicolumn{4}{|l|}{ Design and distance of planting } \\
\hline 1 & Planting design for mangoes square or quincunx & $18(18.00)$ & $\begin{array}{c}20 \\
(20.00)\end{array}$ & $\begin{array}{c}62 \\
(62.00)\end{array}$ \\
\hline 2 & Lay-out follow an east-west orientation & $19(19.00)$ & $\begin{array}{c}22 \\
(22.00)\end{array}$ & $\begin{array}{c}59 \\
(59.00)\end{array}$ \\
\hline 3 & Contour planting on highly sloping areas & $68(68.00)$ & $\begin{array}{c}24 \\
(24.00)\end{array}$ & $8(8.00)$ \\
\hline 4 & Planting distance is $10 \mathrm{mx} 10 \mathrm{~m} 15 \mathrm{mx} 15 \mathrm{~m}$ & $79(79.00)$ & $\begin{array}{c}17 \\
(17.00)\end{array}$ & $4(4.00)$ \\
\hline
\end{tabular}


Table.2 (d) Distribution of the respondents according to adoption of good agriculture practices of cultural management of young trees

\begin{tabular}{|c|c|c|c|c|}
\hline \multirow[t]{2}{*}{ SI. No. } & \multirow[t]{2}{*}{ Practices } & \multicolumn{3}{|c|}{ Adoption $(\mathrm{N}=100)$} \\
\hline & & Complete & Partial & No \\
\hline A. & Early pruning & & & \\
\hline 1 & Pruning is used to reduce the height of trees & $\begin{array}{c}29 \\
(29.00)\end{array}$ & $\begin{array}{c}25 \\
(25.00)\end{array}$ & $46(46.00)$ \\
\hline 2 & $\begin{array}{l}\text { Tree is about } 1 \text { to } 2 \mathrm{~m} \text { height, the terminal portions are } \\
\text { cut/pinched to encourage lateral branching }\end{array}$ & $\begin{array}{c}22 \\
(22.00)\end{array}$ & $\begin{array}{c}27 \\
(27.00)\end{array}$ & $51(51.00)$ \\
\hline 3 & Three to four branches are allowed to grow to $1 \mathrm{~m}$ height & $\begin{array}{c}20 \\
(20.00)\end{array}$ & $\begin{array}{c}22 \\
(22.00)\end{array}$ & $58(58.00)$ \\
\hline 4 & $\begin{array}{l}\text { Second cutting or pruning of terminal portions is done } \\
\text { until the branches are evenly distributed }\end{array}$ & $\begin{array}{c}15 \\
(15.00)\end{array}$ & $\begin{array}{c}29 \\
(29.00)\end{array}$ & $56(56.00)$ \\
\hline 5 & $\begin{array}{l}\text { Tree is top-pruned to control the height and for proper } \\
\text { development of lateral branching for sunlight penetration, } \\
\text { air circulation, and facilitates spraying, bagging and } \\
\text { harvesting operations }\end{array}$ & $\begin{array}{c}45 \\
(45.00)\end{array}$ & $\begin{array}{c}16 \\
(16.00)\end{array}$ & $39(39.00)$ \\
\hline 6 & $\begin{array}{l}\text { Removal of dead, diseased, infested and damaged } \\
\text { branches }\end{array}$ & $\begin{array}{c}87 \\
(87.00)\end{array}$ & $6(6.00)$ & $7(7.00)$ \\
\hline B. & Fertilization management & & & \\
\hline a. & Use mineral fertilizers & & & \\
\hline 1 & $\begin{array}{l}\text { Agricultural lime, magnesium, wood ash, carbonized rice } \\
\text { hull or charcoal as source of carbon, magnesium, } \\
\text { phosphorous, potash and other trace mineral elements }\end{array}$ & $\begin{array}{c}19 \\
(19.00)\end{array}$ & $\begin{array}{c}61 \\
(61.00)\end{array}$ & $20(20.00)$ \\
\hline b. & Use organic fertilizers & & & \\
\hline 1 & $\begin{array}{l}\text { Compost, vermiwash, fermented plant and animal } \\
\text { extracts with molasses as liquid fertilizer for drench and } \\
\text { foliar spray }\end{array}$ & $\begin{array}{c}63 \\
(63.00)\end{array}$ & $\begin{array}{c}37 \\
(37.00)\end{array}$ & -- \\
\hline 2 & Organic foliar fertilizer for spraying & $\begin{array}{c}36 \\
(36.00)\end{array}$ & $\begin{array}{c}41 \\
(41.00)\end{array}$ & $23(23.00)$ \\
\hline 3 & Beneficial Microorganisms for soil improvement & -- & -- & $\begin{array}{c}100 \\
(100.00)\end{array}$ \\
\hline c. & Use inorganic fertilizers $(\mathbf{N}, \mathbf{P}, \mathbf{K})$ & & & \\
\hline 1 & Urea & $\begin{array}{c}29 \\
(29.00)\end{array}$ & - & $71(71.00)$ \\
\hline 2 & SSP & $\begin{array}{c}100 \\
(100.00)\end{array}$ & -- & -- \\
\hline 3 & Potash & $\begin{array}{c}100 \\
(100.00)\end{array}$ & -- & -- \\
\hline d. & Use Micronutrients & & & \\
\hline 1 & Iron & $\begin{array}{c}58 \\
(58.00)\end{array}$ & $\begin{array}{c}18 \\
(18.00)\end{array}$ & $24(24.00)$ \\
\hline 2 & Zinc & $\begin{array}{c}71 \\
(71.00)\end{array}$ & $\begin{array}{c}18 \\
(18.00)\end{array}$ & $11(11.00)$ \\
\hline 3 & Boron & 71 & 18 & $11(11.00)$ \\
\hline
\end{tabular}




\begin{tabular}{|c|c|c|c|c|}
\hline & & $(71.00)$ & $(18.00)$ & \\
\hline 4 & Magnesium & $\begin{array}{c}54 \\
(54.00)\end{array}$ & $\begin{array}{c}22 \\
(22.00)\end{array}$ & $24(24.00)$ \\
\hline 5 & Calcium & $\begin{array}{c}61 \\
(61.00)\end{array}$ & $\begin{array}{c}21 \\
(21.00)\end{array}$ & $18(18.00)$ \\
\hline 6 & $\begin{array}{l}\text { Other } \\
\text { 1. } 19-19-19 \\
\text { 2. } 12-32-16 \\
\text { 3. } 15-15-15\end{array}$ & $\begin{array}{c}78 \\
(78.00)\end{array}$ & $\begin{array}{c}13 \\
(13.00)\end{array}$ & $\begin{array}{c}9 \\
(9.00)\end{array}$ \\
\hline C. & \multicolumn{4}{|l|}{ Irrigation } \\
\hline 1 & Irrigation is done by manual watering & $\begin{array}{c}12 \\
(12.00)\end{array}$ & $\begin{array}{c}16 \\
(16.00)\end{array}$ & $72(72.00)$ \\
\hline 2 & Drip method & $\begin{array}{c}24 \\
(24.00)\end{array}$ & $9(9.00)$ & $67(67.00)$ \\
\hline 3 & Use fertigation equipment & $\begin{array}{c}4 \\
(4.00)\end{array}$ & $2(2.00)$ & $94(94.00)$ \\
\hline 4 & Water plants weekly & $\begin{array}{c}5 \\
(5.00)\end{array}$ & $\begin{array}{c}12 \\
(12.00)\end{array}$ & $83(83.00)$ \\
\hline 5 & Use irrigation schedule & -- & $5(5.00)$ & $95(95.00)$ \\
\hline D. & \multicolumn{4}{|l|}{ Mulching } \\
\hline 1 & $\begin{array}{l}\text { To minimize evaporation, practice mulching using dry } \\
\text { leaves or thick layer of rice hull }(10-15 \mathrm{~cm}) \text { at the base of } \\
\text { the mango tree }\end{array}$ & $\begin{array}{c}58 \\
(58.00)\end{array}$ & $\begin{array}{c}15 \\
(10.00)\end{array}$ & $27(27.00)$ \\
\hline 2 & To prevent growth of weeds and source of organic matter & $\begin{array}{c}47 \\
(47.00)\end{array}$ & $\begin{array}{c}10 \\
(10.00)\end{array}$ & $\begin{array}{c}43 \\
(43.00)\end{array}$ \\
\hline 3 & $\begin{array}{l}\text { Cultivate and break the soil surface up to } 3 \text { inches at the } \\
\text { start of the dry season }\end{array}$ & $\begin{array}{c}12 \\
(12.00)\end{array}$ & $\begin{array}{c}22 \\
(22.00)\end{array}$ & $66(66.00)$ \\
\hline E. & \multicolumn{4}{|l|}{ Drainage } \\
\hline & $\begin{array}{l}\text { On sloping area provide shallow drainage between rows } \\
\text { of trees to drain out excess rain water }\end{array}$ & $\begin{array}{c}7 \\
(7.00)\end{array}$ & $9(9.00)$ & $84(84.00)$ \\
\hline F. & \multicolumn{4}{|l|}{ Inter-cropping } \\
\hline 1 & $\begin{array}{l}\text { Area between the rows of mango trees planted with } \\
\text { vegetables or legumes }\end{array}$ & -- & -- & $\begin{array}{c}100 \\
(100.00)\end{array}$ \\
\hline 2 & $\begin{array}{l}\text { In addition, fruit trees such as papaya, pineapple, or } \\
\text { banana }\end{array}$ & -- & $2(2.00)$ & $98(98.00)$ \\
\hline G. & \multicolumn{4}{|l|}{ Weed control } \\
\hline 1 & Ring cultivation, about $1 \mathrm{~m}$ radius from the trunk & $\begin{array}{c}63 \\
(63.00)\end{array}$ & $\begin{array}{c}14 \\
(14.00)\end{array}$ & $23(23.00)$ \\
\hline 2 & Inter-row cultivation (plough / tractor/ grass cutter) & -- & $\begin{array}{c}17 \\
(17.00)\end{array}$ & $\begin{array}{c}83 \\
(83.00)\end{array}$ \\
\hline 3 & Cover cropping with leguminous creeping vines & -- & -- & $\begin{array}{c}100 \\
(100.00)\end{array}$ \\
\hline 4 & Use of herbicides & $\begin{array}{c}6 \\
(6.00)\end{array}$ & $\begin{array}{c}22 \\
(22.00)\end{array}$ & $72(72.00)$ \\
\hline
\end{tabular}


Table.2 (e) Distribution of the respondents according to adoption of good agriculture practices of cultural management of bearing trees

\begin{tabular}{|c|c|c|c|c|}
\hline \multirow{2}{*}{$\begin{array}{l}\text { Sl. } \\
\text { No. }\end{array}$} & \multirow[t]{2}{*}{ Practices } & \multicolumn{3}{|c|}{ Adoption $(\mathrm{N}=100)$} \\
\hline & & Complete & Partial & No \\
\hline A. & \multicolumn{4}{|l|}{ Pruning } \\
\hline 1 & $\begin{array}{l}\text { Removal of unnecessary branches such as water sprouts, } \\
\text { dried, disease-infected branches and all branches that grow } \\
\text { crowding the canopy }\end{array}$ & $\begin{array}{c}70 \\
(70.00)\end{array}$ & $\begin{array}{c}22 \\
(22.00)\end{array}$ & $8(8.00)$ \\
\hline 2 & Pruning done during summer months or dry and warm days & $\begin{array}{c}19 \\
(19.00)\end{array}$ & $\begin{array}{c}11 \\
(11.00)\end{array}$ & $\begin{array}{c}70 \\
(70.00)\end{array}$ \\
\hline B. & \multicolumn{4}{|l|}{ Flower induction } \\
\hline \multirow[t]{4}{*}{1} & $\begin{array}{l}\text { Prior to spraying or smudging, the leaves of trees are checked } \\
\text { for attributes of readiness to flower ie. }\end{array}$ & & & \\
\hline & i. Age of the leaves at least 7 months from flushing & $\begin{array}{c}13 \\
(13.00)\end{array}$ & $\begin{array}{c}26 \\
(26.00)\end{array}$ & $\begin{array}{c}61 \\
(61.00)\end{array}$ \\
\hline & ii. Leaves are dark green, coppery and brittle & $\begin{array}{c}20 \\
(20.00)\end{array}$ & $\begin{array}{c}35 \\
(35.00)\end{array}$ & $\begin{array}{c}45 \\
(45.00)\end{array}$ \\
\hline & iii. Buds are prominent and dormant & $\begin{array}{c}25 \\
(25.00)\end{array}$ & $\begin{array}{c}39 \\
(39.00)\end{array}$ & $\begin{array}{c}36 \\
(36.00)\end{array}$ \\
\hline 2 & \multicolumn{4}{|l|}{ Use flower inducers } \\
\hline & 1. Potassium nitrate $\left(\mathrm{KNO}_{3}\right)$-based flower inducers & $\begin{array}{c}3 \\
(3.00)\end{array}$ & $\begin{array}{c}10 \\
(10.00)\end{array}$ & $\begin{array}{c}87 \\
(87.00)\end{array}$ \\
\hline & 2. Sodium nitrate $\left(\mathrm{NaNO}_{3}\right)$ & $\begin{array}{c}5 \\
(5.00)\end{array}$ & $8(8.00)$ & $\begin{array}{c}87 \\
(87.00)\end{array}$ \\
\hline & 3. Liquid ammonium nitrate $\left(\mathrm{NH}_{4} \mathrm{NO}_{3}\right)$ & $\begin{array}{c}4 \\
(4.00)\end{array}$ & $3(3.00)$ & $\begin{array}{c}93 \\
(93.00)\end{array}$ \\
\hline & 4. Calcium ammonium nitrate $\left(\mathrm{CaNH}_{4} \mathrm{NO}_{3}\right)$ & $\begin{array}{c}9 \\
(9.00)\end{array}$ & $\begin{array}{c}19 \\
(19.00)\end{array}$ & $\begin{array}{c}72 \\
(72.00)\end{array}$ \\
\hline & 5. Calcium nitrate $\left[\mathrm{Ca}\left(\mathrm{NO}_{3}\right)_{2}\right]$ & $\begin{array}{c}9 \\
(9.00)\end{array}$ & $\begin{array}{c}36 \\
(36.00)\end{array}$ & $\begin{array}{c}55 \\
(55.00)\end{array}$ \\
\hline & 6. Ethylene (Ethryle) & $\begin{array}{c}7 \\
(7.00)\end{array}$ & $\begin{array}{c}34 \\
(34.00)\end{array}$ & $\begin{array}{c}59 \\
(59.00)\end{array}$ \\
\hline C. & \multicolumn{4}{|l|}{ Flowering and fruit management } \\
\hline 1 & $\begin{array}{l}\text { Foliar application of liquid fertilizer at } 18-25 \text { days after } \\
\text { flower induction (DAFI) }\end{array}$ & $\begin{array}{c}23 \\
(23.00)\end{array}$ & $\begin{array}{c}33 \\
(33.00)\end{array}$ & $\begin{array}{c}44 \\
(44.00)\end{array}$ \\
\hline 2 & $\begin{array}{l}\text { Pollinating insects like blue flies \& bees are encouraged by } \\
\text { avoiding spraying of pesticides during full bloom }\end{array}$ & $\begin{array}{c}17 \\
(17.00)\end{array}$ & $\begin{array}{c}57 \\
(57.00)\end{array}$ & $\begin{array}{c}26 \\
(26.00)\end{array}$ \\
\hline 3 & $\begin{array}{l}\text { Five percent }(5 \%) \text { sugar or honey solution is sprayed as spot } \\
\text { application to attract pollinators during full bloom }\end{array}$ & -- & $\begin{array}{c}13 \\
(13.00)\end{array}$ & $\begin{array}{c}87 \\
(87.00)\end{array}$ \\
\hline 4 & $\begin{array}{l}\text { Branches can be shaken after a rain or early in the morning } \\
\text { during blooming to remove flowers affected by disease }\end{array}$ & -- & $\begin{array}{c}40 \\
(40.00)\end{array}$ & $\begin{array}{c}60 \\
(60.00)\end{array}$ \\
\hline 5 & $\begin{array}{l}\text { Trees are irrigated weekly, during summer dry months } \\
\text { approximately } 100-300 \mathrm{~L} \text { of water per tree per week }\end{array}$ & -- & $\begin{array}{c}12 \\
(12.00)\end{array}$ & $\begin{array}{c}88 \\
(88.00)\end{array}$ \\
\hline 6 & Bagging is done at 55 to $60 \mathrm{DAFI}$ & -- & $8(8.00)$ & $\begin{array}{c}92 \\
(92.00)\end{array}$ \\
\hline
\end{tabular}


Table.2 (f) Distribution of the respondents according to adoption of good agriculture practices of integrated pest management

\begin{tabular}{|c|c|c|c|c|}
\hline \multirow{2}{*}{$\begin{array}{l}\text { Sl. } \\
\text { No. }\end{array}$} & \multirow[t]{2}{*}{ Practices } & \multicolumn{3}{|c|}{ Adoption $(\mathrm{N}=100)$} \\
\hline & & Complete & Partial & No \\
\hline a. & Ants & & & \\
\hline 1 & $\begin{array}{l}\text { Prune the mango tree and remove all unnecessary } \\
\text { branches that provide favourable environment for } \\
\text { ants }\end{array}$ & $\begin{array}{c}16 \\
(16.00)\end{array}$ & $15(15.00)$ & $69(69.00)$ \\
\hline 2 & Apply insecticides during heavy infestation & $100(100.00)$ & -- & -- \\
\hline b. & Fruit fly & & & \\
\hline 1 & $\begin{array}{l}\text { Collect the infested fruits and burry deep into the } \\
\text { soil to prevent the insect from completing its life } \\
\text { cycle }\end{array}$ & $\begin{array}{c}70 \\
(70.00)\end{array}$ & $16(16.00)$ & $14(14.00)$ \\
\hline 2 & Bag the fruits with appropriate bagging materials & -- & $\begin{array}{c}8 \\
(8.00)\end{array}$ & $92(92.00)$ \\
\hline 3 & Use 'Rakshak' traps & $\begin{array}{c}80 \\
(80.00)\end{array}$ & $13(13.00)$ & $\begin{array}{c}7 \\
(7.00)\end{array}$ \\
\hline 4 & $\begin{array}{l}\text { Harvest fruits at mature green stage since fruit flies } \\
\text { are attracted to them as soon as their surfaces } \\
\text { become yellow }\end{array}$ & $\begin{array}{c}42 \\
(42.00)\end{array}$ & $\begin{array}{c}7 \\
(7.00)\end{array}$ & $51(51.00)$ \\
\hline c. & Mango thrips & & & \\
\hline 1 & $\begin{array}{l}\text { Prune or cut off excess branches to improve } \\
\text { aeration and to allow more light }\end{array}$ & $\begin{array}{c}16 \\
(16.00)\end{array}$ & $10(10.00)$ & $\begin{array}{c}74 \\
(74.00)\end{array}$ \\
\hline 2 & Spray registered insecticides as a fine mist & $100(100.00)$ & -- & -- \\
\hline d. & Mango leafhopper & & & \\
\hline 1 & $\begin{array}{l}\text { Light trapping before flower induction to reduce } \\
\text { initial leaf hopper population }\end{array}$ & $\begin{array}{c}8 \\
(8.00)\end{array}$ & $\begin{array}{c}2 \\
(2.00)\end{array}$ & $90(90.00)$ \\
\hline 2 & Use of insecticides & $100(100.00)$ & -- & -- \\
\hline 3 & $\begin{array}{l}\text { Avoid excessive application of fungicides to } \\
\text { conserve beneficial fungi that attack the leafhopper }\end{array}$ & $\begin{array}{c}50 \\
(50.00)\end{array}$ & $47(47.00)$ & $\begin{array}{c}3 \\
(3.00)\end{array}$ \\
\hline 4 & $\begin{array}{l}\text { Apply insecticides only when there are at least } \\
\text { three leafhoppers per panicle }\end{array}$ & $\begin{array}{c}67 \\
(67.00)\end{array}$ & $23(23.00)$ & $10(10.00)$ \\
\hline
\end{tabular}

Table.2 (g) Distribution of the respondents according to adoption of good agriculture practices of integrated disease management

\begin{tabular}{|c|l|c|c|c|}
\hline Sl. No. & \multicolumn{1}{|c|}{ Practices } & \multicolumn{2}{|c|}{ Adoption (N=100) } \\
\cline { 3 - 5 } & \multicolumn{2}{|c|}{ Complete } & Partial & No \\
\hline A. & Anthracnose & $100(100.00)$ & -- & - \\
\hline $\mathbf{1}$ & Spray fungicides & $91(91.00)$ & $7(7.00)$ & $2(2.00)$ \\
\hline $\mathbf{2}$ & Use spray schedule & $94(94.00)$ & $4(4.00)$ & $2(2.00)$ \\
\hline B. & Powdery Mildew & $12(12.00)$ & $5(5.00)$ & $83(83.00)$ \\
\hline $\mathbf{1}$ & Use spray schedule & & & \\
\hline $\mathbf{2}$ & Use of sulphur smoke & & & \\
\hline
\end{tabular}


Table.2 (h) Distribution of the respondents according to adoption of good agriculture practices of pesticide management

\begin{tabular}{|c|c|c|c|c|}
\hline \multirow{2}{*}{$\begin{array}{c}\text { Sl. } \\
\text { No. }\end{array}$} & \multirow[t]{2}{*}{ Practices } & \multicolumn{3}{|c|}{ Adoption (N=100) } \\
\hline & & Complete & Partial & No \\
\hline A. & \multicolumn{4}{|c|}{ Good Agricultural Practices (GAP) pertaining to the use of pesticides: } \\
\hline 1 & Use registered pesticides & $100(100.00)$ & -- & -- \\
\hline \multirow[t]{10}{*}{2} & Read and follow the labelled instructions & -- & -- & -- \\
\hline & a. Product information & & & \\
\hline & i. Active ingredient(s) & $100(100.00)$ & -- & -- \\
\hline & ii. Solvent & $100(100.00)$ & -- & -- \\
\hline & b. Directions for use & & & \\
\hline & i. Crops & $\begin{array}{c}98 \\
(98.00)\end{array}$ & $\begin{array}{c}2 \\
(2.00)\end{array}$ & -- \\
\hline & ii. Target pests & $100(100.00)$ & -- & -- \\
\hline & iii. Dosage, frequency and timing of application & $100(100.00)$ & -- & -- \\
\hline & iv. Pre-cautionary measures & $\begin{array}{c}98 \\
(98.00)\end{array}$ & $\begin{array}{c}2 \\
(2.00)\end{array}$ & -- \\
\hline & v. Storage and disposal & $\begin{array}{c}91 \\
(91.00)\end{array}$ & $\begin{array}{c}9 \\
(9.00)\end{array}$ & -- \\
\hline 3 & First aid and medical treatment in case of poisoning & $\begin{array}{c}83 \\
(83.00)\end{array}$ & $17(17.00)$ & -- \\
\hline 4 & Emergency contact number & $100(100.00)$ & -- & -- \\
\hline B. & \multicolumn{4}{|c|}{ Good agriculture practices for the proper use and handling before mixing } \\
\hline a. & \multicolumn{4}{|l|}{ Before mixing } \\
\hline 1 & Keep spraying equipment in good condition & $100(100.00)$ & -- & -- \\
\hline 2 & Check sprayer for defects & $100(100.00)$ & -- & -- \\
\hline 3 & $\begin{array}{l}\text { Maintain a record of maintenance check-up of } \\
\text { spraying equipment }\end{array}$ & $\begin{array}{c}96 \\
(96.00)\end{array}$ & -- & $\begin{array}{c}4 \\
(4.00)\end{array}$ \\
\hline 4 & Clean the nozzles with water or a soft-probing device & $100(100.00)$ & -- & -- \\
\hline 5 & $\begin{array}{l}\text { Use of appropriate personal protective equipment } \\
\text { (PPEs) used, read the product label }\end{array}$ & $100(100.00)$ & -- & -- \\
\hline b. & \multicolumn{4}{|l|}{ During mixing } \\
\hline 1 & $\begin{array}{l}\text { Use a measuring cup or graduated cylinder in } \\
\text { measuring the concentrated formulated pesticide }\end{array}$ & $100(100.00)$ & -- & -- \\
\hline 2 & $\begin{array}{l}\text { Use clean water for mixing pesticides to avoid } \\
\text { microbial contamination of the mango fruits }\end{array}$ & $100(100.00)$ & -- & -- \\
\hline 3 & $\begin{array}{l}\text { Contents of the pesticide bottle are used up, rinse the } \\
\text { bottle } 3 \text { times with water and pour into the last sprayer } \\
\text { tank load }\end{array}$ & $100(100.00)$ & -- & -- \\
\hline 4 & Use appropriate gloves to minimize dermal exposure & $\begin{array}{c}87 \\
(87.00)\end{array}$ & $13(13.00)$ & -- \\
\hline c. & \multicolumn{4}{|l|}{ During application } \\
\hline 1 & $\begin{array}{l}\text { Wear the necessary protection cloths i.e. cover nose } \\
\text { and mouth, wear long-sleeved shirts and long pants }\end{array}$ & $\begin{array}{c}95 \\
(95.00)\end{array}$ & $\begin{array}{c}5 \\
(5.00)\end{array}$ & -- \\
\hline 2 & Spray when wind is slow & 82 & $18(18.00)$ & -- \\
\hline
\end{tabular}




\begin{tabular}{|c|c|c|c|c|}
\hline & & $(82.00)$ & & \\
\hline 3 & $\begin{array}{l}\text { Spray inner canopy first before spraying the outer } \\
\text { canopy }\end{array}$ & $100(100.00)$ & -- & -- \\
\hline 4 & Use a power sprayer with an extended boom & $100(100.00)$ & -- & -- \\
\hline 5 & $\begin{array}{l}\text { Maintain a record of spray application indicating } \\
\text { information of pesticide used, volume used, area } \\
\text { sprayed, and operator }\end{array}$ & $\begin{array}{c}95 \\
(95.00)\end{array}$ & $\begin{array}{c}5 \\
(5.00)\end{array}$ & -- \\
\hline d. & After spraying & & & \\
\hline 1 & $\begin{array}{l}\text { Clean spray equipment by flushing the remaining } \\
\text { pesticide solution using detergent and clean water }\end{array}$ & $100(100.00)$ & -- & -- \\
\hline 2 & $\begin{array}{l}\text { Dispose contaminated water or rinsed outside the } \\
\text { waterways }\end{array}$ & $100(100.00)$ & -- & -- \\
\hline 3 & Change working clothes immediately after spraying & $100(100.00)$ & -- & -- \\
\hline 4 & Wash your hands with soap and water & $100(100.00)$ & -- & -- \\
\hline 5 & Soak clothes in water and detergent & $100(100.00)$ & -- & -- \\
\hline C. & Good agriculture practices for storage and disposal & & & \\
\hline a. & Storage & & & \\
\hline $\mathbf{1}$ & Store pesticides in their original labelled containers & $\begin{array}{c}97 \\
(97.00)\end{array}$ & $\begin{array}{c}3 \\
(3.00)\end{array}$ & -- \\
\hline 2 & $\begin{array}{l}\text { Keep pesticides locked in a storeroom and out of } \\
\text { children's reach }\end{array}$ & $100(100.00)$ & -- & -- \\
\hline 3 & $\begin{array}{l}\text { Keep pesticides away from fire or open flame, stove or } \\
\text { lamps }\end{array}$ & $100(100.00)$ & -- & -- \\
\hline 4 & $\begin{array}{l}\text { Partially used pesticide bottles must be placed inside a } \\
\text { thick plastic bag to avoid hand contamination }\end{array}$ & $100(100.00)$ & -- & -- \\
\hline b. & Disposal & & & \\
\hline 1 & $\begin{array}{l}\text { Dispose of empty pesticide bottles and cartons into a } \\
\text { pesticide disposal pit }\end{array}$ & $\begin{array}{c}100 \\
(100.00)\end{array}$ & -- & -- \\
\hline 2 & $\begin{array}{l}\text { Dig disposal pit in an area away from people and } \\
\text { animals, and far from water sources }\end{array}$ & $100(100.00)$ & -- & -- \\
\hline D. & Good agricultural practices of insect resistance mana & ement & & \\
\hline 1 & Use products according to the recommended dosage & $\begin{array}{c}100 \\
(100.00)\end{array}$ & -- & -- \\
\hline 2 & $\begin{array}{l}\text { Use appropriate, well-maintained equipment to apply } \\
\text { insecticides / fungicides }\end{array}$ & $100(100.00)$ & -- & -- \\
\hline 3 & $\begin{array}{l}\text { Use recommended water volume and spray pressure in } \\
\text { order to obtain optimal coverage of the canopy }\end{array}$ & $100(100.00)$ & -- & -- \\
\hline 4 & $\begin{array}{l}\text { Target the pests at their vulnerable stage, (young } \\
\text { instars, larvae) where possible }\end{array}$ & $100(100.00)$ & -- & -- \\
\hline 5 & $\begin{array}{l}\text { Use appropriate economic thresholds level and spray } \\
\text { intervals }\end{array}$ & $100(100.00)$ & -- & -- \\
\hline 6 & $\begin{array}{l}\text { Use alternately products of different modes of action } \\
\text { or from different chemical groups }\end{array}$ & $100(100.00)$ & -- & -- \\
\hline 7 & Mix different pesticides & $\begin{array}{c}84 \\
(84.00)\end{array}$ & $12(12.00)$ & $\begin{array}{c}4 \\
(4.00)\end{array}$ \\
\hline
\end{tabular}


Table.2 (i) Distribution of the respondents according to adoption of good agriculture practices of harvesting and post-harvesting management technology

\begin{tabular}{|c|c|c|c|c|}
\hline \multirow{2}{*}{$\begin{array}{l}\text { Sl. } \\
\text { No. }\end{array}$} & \multirow[t]{2}{*}{ Practices } & \multicolumn{3}{|c|}{ Adoption $(\mathrm{N}=100)$} \\
\hline & & Complete & Partial & No \\
\hline A. & Maturity indices & & & \\
\hline 1 & $\begin{array}{l}\text { Fruits harvested upon reaching maturity of 105-115 } \\
\text { DAFI (late flower induction - January, February, } \\
\text { March) }\end{array}$ & $\begin{array}{c}53 \\
(53.00)\end{array}$ & $33(33.00)$ & $\begin{array}{c}14 \\
(14.00)\end{array}$ \\
\hline 2 & $\begin{array}{l}\text { 120-130 DAFI (early induction - October, November, } \\
\text { December). }\end{array}$ & $\begin{array}{c}53 \\
(53.00)\end{array}$ & $31(31.00)$ & $\begin{array}{c}16 \\
(16.00)\end{array}$ \\
\hline 3 & Flattening or expansion of shoulders & $\begin{array}{c}92 \\
(92.00)\end{array}$ & $\begin{array}{c}08 \\
(8.00)\end{array}$ & -- \\
\hline 4 & Presence of "bloom" or powdery deposit on the fruit & $\begin{array}{c}58 \\
(58.00)\end{array}$ & $11(11.00)$ & $\begin{array}{c}31 \\
(31.00)\end{array}$ \\
\hline 5 & Yellow green colour near pedicel & $\begin{array}{c}30 \\
(30.00)\end{array}$ & $\begin{array}{c}08 \\
(8.00)\end{array}$ & $\begin{array}{c}62 \\
(62.00)\end{array}$ \\
\hline 6 & $\begin{array}{l}\text { Floatation of fruits in } 1 \% \text { salt solution }(100 \mathrm{~g} \text { salt } / 10 \mathrm{~L} \\
\text { of water) }\end{array}$ & -- & $\begin{array}{c}4 \\
(4.00)\end{array}$ & $\begin{array}{c}96 \\
(96.00)\end{array}$ \\
\hline 7 & Use physical method & $\begin{array}{c}48 \\
(48.00)\end{array}$ & $38(38.00)$ & $\begin{array}{c}14 \\
(14.00)\end{array}$ \\
\hline B. & Harvesting & & & \\
\hline 1 & Harvesters must wash their hands before harvesting & $\begin{array}{c}70 \\
(70.00)\end{array}$ & $11(11.00)$ & $\begin{array}{c}19 \\
(19.00)\end{array}$ \\
\hline 2 & $\begin{array}{l}\text { Good personal hygiene practice to avoid cross- } \\
\text { contamination of the produce }\end{array}$ & $\begin{array}{c}81 \\
(81.00)\end{array}$ & $\begin{array}{c}19 \\
(19.00)\end{array}$ & -- \\
\hline 3 & $\begin{array}{l}\text { Fruits harvested between 9:00 am and 3:00 pm for } \\
\text { lesser latex flow }\end{array}$ & $\begin{array}{c}50 \\
(50.00)\end{array}$ & $34(34.00)$ & $\begin{array}{c}16 \\
(16.00)\end{array}$ \\
\hline 4 & $\begin{array}{l}\text { Leave about } 2.0 \mathrm{~cm} \text { pedicel on the fruit to minimize } \\
\text { latex flow which may burn the fruit }\end{array}$ & $100(100.00)$ & -- & -- \\
\hline 5 & $\begin{array}{l}\text { Minimize damage and contamination of fruits with } \\
\text { soil, pathogens, fertilizers or other agro-chemicals }\end{array}$ & $\begin{array}{c}89 \\
(89.00)\end{array}$ & $\begin{array}{c}11 \\
(11.00)\end{array}$ & -- \\
\hline 6 & $\begin{array}{l}\text { Harvested fruits kept away from direct sunlight and } \\
\text { brought immediately to the shade or packing house for } \\
\text { sorting and other post-harvest activities }\end{array}$ & $100(100.00)$ & -- & -- \\
\hline 7 & $\begin{array}{l}\text { Harvesting crates/containers (e.g. bamboo baskets, } \\
\text { stackable plastic crates, etc.) regularly cleaned and } \\
\text { maintained }\end{array}$ & $100(100.00)$ & -- & -- \\
\hline 8 & $\begin{array}{l}\text { Containers of harvested fruits clearly identified with } \\
\text { names or codes containing date of harvest, time, block } \\
\text { number, volume and name of harvester/farmer }\end{array}$ & $\begin{array}{c}98 \\
(98.00)\end{array}$ & $\begin{array}{c}02 \\
(2.00)\end{array}$ & -- \\
\hline C. & Post-harvest handling & & & \\
\hline 1 & $\begin{array}{l}\text { Harvested fruits sorted/packed on-farm or transported } \\
\text { to a common packinghouse facility }\end{array}$ & $100(100.00)$ & -- & -- \\
\hline 2 & $\begin{array}{l}\text { Fruits to be sorted out and placed on paper in } \\
\text { packinghouse to avoid cross-contamination of the } \\
\text { produce }\end{array}$ & $100(100.00)$ & -- & -- \\
\hline 3 & $\begin{array}{l}\text { Sorters/packers wash their hands with soap or detergent } \\
\text { before handling the produce }\end{array}$ & $100(100.00)$ & -- & -- \\
\hline
\end{tabular}




\begin{tabular}{|c|c|c|c|c|}
\hline 4 & $\begin{array}{l}\text { For packinghouse facilities, the packing room separated } \\
\text { from toilet facilities and kept clean, tidy, well } \\
\text { ventilated and free of foul smell at all times }\end{array}$ & $100(100.00)$ & -- & -- \\
\hline 5 & Mangoes sorted according to size and quality & $100(100.00)$ & -- & -- \\
\hline 6 & $\begin{array}{l}\text { Sorted and graded mangoes should be inspected and } \\
\text { trimmed of stems connected to the fruits }\end{array}$ & $100(100.00)$ & -- & -- \\
\hline 7 & $\begin{array}{l}\text { Fruits washed clean with a mixture of potable water } \\
\text { and mild soap }\end{array}$ & $100(100.00)$ & -- & -- \\
\hline D. & \multicolumn{4}{|l|}{ Post-harvest treatments: } \\
\hline a. & \multicolumn{4}{|l|}{ Hot water treatment (HWT) } \\
\hline 1 & $\begin{array}{l}\text { Newly harvested fruits are dipped in warm water } \\
\text { (about } 52-55^{\circ} \mathrm{C} \text { ) for about } 10 \mathrm{~min}\end{array}$ & -- & -- & -- \\
\hline 2 & $\begin{array}{l}\text { Fruits are then rinsed in cool running water for } 10 \mathrm{~min} \\
\text { (hydro-cooling), air-dried and packed }\end{array}$ & -- & -- & -- \\
\hline \multirow[t]{2}{*}{ b. } & \multicolumn{4}{|l|}{ Rapid hot water treatment } \\
\hline & $\begin{array}{l}\text { Fruits are submerged in hot water }\left(60^{\circ} \mathrm{C}\right) \text { for } 30 \mathrm{sec} \text { to } \\
\text { one (1) } \mathrm{min}\end{array}$ & -- & -- & -- \\
\hline c. & \multicolumn{4}{|l|}{ Extendedhot water treatment } \\
\hline 1 & $\begin{array}{l}\text { Fruits are submerged in hot water }\left(46 \text { or } 47^{\circ} \mathrm{C} \text { ) for two }\right. \\
\text { (2) hours }\end{array}$ & -- & -- & -- \\
\hline 2 & $\begin{array}{l}\text { Temperature is maintained for } 15 \mathrm{~min} \text { at } 46^{\circ} \mathrm{C} \text { or } 10 \\
\text { min at } 47^{\circ} \mathrm{C}\end{array}$ & -- & -- & -- \\
\hline 3 & Fruits are then allowed to cool by air-drying & -- & -- & -- \\
\hline \multirow[t]{2}{*}{ d. } & \multicolumn{4}{|l|}{ Vapour heat treatment } \\
\hline & $\begin{array}{l}\text { Fruits are heated in a chamber with vapour-saturated } \\
\text { air until the pulp reaches a temperature of } 46^{\circ} \mathrm{C} \text { which } \\
\text { is maintained for } 10 \mathrm{~min}\end{array}$ & -- & -- & -- \\
\hline e. & \multicolumn{4}{|l|}{ Packaging } \\
\hline 1 & $\begin{array}{l}\text { Mangoes sorted, washed and subjected to post-harvest } \\
\text { treatment(s) are then packed in suitable packaging } \\
\text { containers }\end{array}$ & $\begin{array}{c}98 \\
(98.00)\end{array}$ & $\begin{array}{c}02 \\
(2.00)\end{array}$ & -- \\
\hline \multirow[t]{7}{*}{2} & $\begin{array}{l}\text { Each packaging container clearly labelled with the } \\
\text { following information }\end{array}$ & & & \\
\hline & i. Mango grower's accreditation code & $100(100.00)$ & -- & -- \\
\hline & ii. Name of commodity & $100(100.00)$ & -- & -- \\
\hline & iii. Net weight & $100(100.00)$ & -- & -- \\
\hline & iv. Batch number and date of packing & $100(100.00)$ & -- & -- \\
\hline & $\begin{array}{l}\text { v. Name of Plant Quarantine Service (PQS) signing } \\
\text { officer }\end{array}$ & $\begin{array}{c}14 \\
(14.00)\end{array}$ & -- & $\begin{array}{c}86 \\
(86.00)\end{array}$ \\
\hline & vi. Name of brand (Ratnagiri Hapus) & $100(100.00)$ & -- & -- \\
\hline f. & \multicolumn{4}{|l|}{ Storage facilities for fruits } \\
\hline $\mathbf{1}$ & $\begin{array}{l}\text { Storage facilities sanitized and free from decaying plant } \\
\text { waste and foul smell }\end{array}$ & $\begin{array}{c}74 \\
(74.00)\end{array}$ & $\begin{array}{c}05 \\
(5.00)\end{array}$ & $\begin{array}{c}21 \\
(21.00)\end{array}$ \\
\hline 2 & $\begin{array}{l}\text { Packed mangoes stored in a cold room (temperature } \\
12-14^{\circ} \mathrm{C} \text {; relative humidity } 85-95 \% \text { ) immediately after } \\
\text { packing }\end{array}$ & $\begin{array}{c}43 \\
(43.00)\end{array}$ & $\begin{array}{c}05 \\
(5.00)\end{array}$ & $\begin{array}{c}52 \\
(52.00)\end{array}$ \\
\hline 3 & Cooling equipment cleaned and inspected frequently & $\begin{array}{c}45 \\
(45.00)\end{array}$ & $\begin{array}{c}03 \\
(3.00)\end{array}$ & $\begin{array}{c}52 \\
(52.00)\end{array}$ \\
\hline
\end{tabular}


Table.2 (j) Distribution of the respondents according to adoption of good agriculture practices of farm management

\begin{tabular}{|c|c|c|c|c|}
\hline \multirow{2}{*}{$\begin{array}{l}\text { Sl. } \\
\text { No. }\end{array}$} & \multirow[t]{2}{*}{ Practices } & \multicolumn{3}{|c|}{ Adoption $(\mathrm{N}=100)$} \\
\hline & & Complete & Partial & No \\
\hline $\mathbf{1}$ & All records updated and kept up to two years & $\begin{array}{c}73 \\
(73.00)\end{array}$ & $27(27.00)$ & -- \\
\hline 2 & $\begin{array}{l}\text { Copies of laboratory analysis and certificates } \\
\text { that verify compliance with this code must be } \\
\text { filed }\end{array}$ & $\begin{array}{c}90 \\
(90.00)\end{array}$ & $10(10.00)$ & -- \\
\hline 3 & $\begin{array}{l}\text { Records of lot number must be maintained for all } \\
\text { produce leaving the farm }\end{array}$ & $\begin{array}{c}97 \\
(97.00)\end{array}$ & $\begin{array}{c}03 \\
(3.00)\end{array}$ & -- \\
\hline 4 & Staff training records also be maintained & $\begin{array}{c}78 \\
(78.00)\end{array}$ & $\begin{array}{c}04 \\
(4.00)\end{array}$ & $\begin{array}{c}18 \\
(18.00)\end{array}$ \\
\hline 5 & First aid kit available on farm & $100(100.00)$ & -- & -- \\
\hline 6 & Record of orchard/field /plot/number & $100(100.00)$ & -- & -- \\
\hline 7 & $\begin{array}{l}\text { Records of application of pesticides or } \\
\text { insecticides }\end{array}$ & $100(100.00)$ & -- & -- \\
\hline 8 & $\begin{array}{l}\text { Location, date of application, trained person } \\
\text { assigned and maintenance properly recorded }\end{array}$ & $\begin{array}{c}98 \\
(98.00)\end{array}$ & $\begin{array}{c}02 \\
(2.00)\end{array}$ & -- \\
\hline
\end{tabular}

\section{Good agriculture practices of pesticide management}

The data with regard to the adoption of good agriculture practices of pesticide management by the mango growers are presented in Table 2 (h).

It is revealed from Table $2(\mathrm{~h})$ that among the good agriculture practices of pesticide management, cent per cent (100.00 per cent) of the respondents had 'complete' adoption of 'use registered pesticides' and 'read and follow the label instructions' in case 'product information' i.e. active ingredients and solvent. In case directions for use, it is observed that near to cent per cent or cent per cent of the respondents had 'complete' adoption of 'crops', 'target pest' and 'dosage, frequency and timing of application', 'precautionary measures' and 'storage and disposal'. Whereas, 'first aid and medical treatment in case of poisoning' (83.00 per cent) and 'emergency contact number' (100.00 per cent) had 'complete' adoption.
Good agriculture practices of harvesting and post-harvesting management technology

The data with regard to the adoption of good agriculture practices of harvesting and postharvesting management technology by the mango growers are presented in Table 2 (i).

It is noticed from Table 2 (i) that with regards to maturity indices, more than four-fifth (92.00 per cent) of the respondents had adopted 'flattening or expansion of shoulders' 'completely', whereas majority of the respondents had adopted 'fruits harvested upon reaching maturity of 105-115 DAFI (late flower induction - January, February, March)' 'completely', '120-130 DAFI (early induction - October, November, December)', "presence of "bloom" or powdery deposit on the fruit' and 'use physical method'. Whereas it is observed that more than three-fifth (62.00 per cent) of the respondents did not adopt 'yellow green colour near pedicel' practice and more than four-fifth (96.00 per cent) of 
the respondents did not adopt 'floatation of fruits in $1 \%$ salt solution $(100 \mathrm{~g}$ salt/10L of water)'.

\section{Good agriculture practices of farm management}

The data with regard to the adoption of good agriculture practices of farm management by the mango growers are presented in Table 2 (j).

It is noticed from Table $2(\mathrm{j})$ that near to cent per cent and cent per cent of the respondents had 'completely' adopted 'first aid kit available on farm', 'record of orchard/field /plot/number' and 'records of application of pesticides or insecticides', 'location, date of application and a trained person assigned and maintenance properly recorded' and 'records of lot number must be maintained for all produce leaving the farm'. Whereas, majority of the respondents had 'completely' adopted practice 'copies of laboratory analysis and certificates that verify compliance with this code must be filed', 'all records updated and kept up to two years' and 'staff training records also be maintained'

In conclusion mango has become a cash crop for the farmers of the Konkan region. Efforts are being made at different levels to maximize the area, production and productivity of mango in Konkan region. The GLOBAL GAP standard is primarily designed to reassure the consumers about how food is produced on the farm by minimizing detrimental environmental impacts of farming operations, reducing the use of chemical inputs and ensuring a responsible approach to worker health and safety, as well as, animal welfare. GAP is a series of principles, rules and technical recommendations, with the aim of providing a safe product for direct consumption or industrial processing. Considering the scope and opportunity in the world market, there is a need to give importance to quality assurance of mango fruits. So also, there is a need to keep quality, hygienic conditions and standard residue control, so that the fruits qualify all analytical tests. The findings of the study pertaining to personal, socio-economic, communicational and psychological characteristics of the mango growers may help the agricultural development agencies to identify the prospective mango growers and to plan out strategy to speed up the adoption of GAP in mango cultivation. The study pointed out that the mango growers had differential attitude about GAP certification for mango. The concerned organizations and personnel need to intensify the efforts in that direction. It also suggests that only possessing favourable attitude about GAP certification is not enough to promote adoption and induce positive perception about GAP among the mango growers. But, it is necessary to give the actual experience with hands on training, support of Government for certification and provide them services to practice GAP at their field condition.

\section{References}

Hobbs, J. E. (2003). Incentives for the adoption of good Agricultural Practices (GAPs). FAO consultation on Good Agricultural Practices, Rome. 1-26.

Jadhav, B. (2009).Technological Gap in adoption of recommended practices of mango cultivation. M.Sc. (Agri.) Thesis. University of Agricultural Sciences. Dharwad.

Karan Singh, Verma, S. R., Sharma, F. L. and B. Upadhyay. (2010). Adoption of improved mango cultivation technology among the farmers of Southern Rajasthan. Rajasthan Journal of Extension Education. 19: 128-132.

Kota, S. K., Tarde, V. J. and Babar, M. S. 
(2012). Knowledge and adoption of export oriented practices followed by the mango growers. International Journal of Extension Education. 8: 51 55.

Mahadik, R. P., Mehta, P. G and Patil, V. G. (2008). Adoption of recommended mango cultivation technology by mango growers. Karnataka Journal of Agricultural Science. 21(2): (314-315) 2008.

Manvar, V. S. (1999). A study on adoption of recommended package of practices of mango by mango growers in Aurangabad district. M.Sc. (Agri.) Thesis. Marathwada Agricultural University, Parbhani.

Modi, R. D. (2010). A study on knowledge and adoption of post-harvest management practices among the mango growers of northern Karnataka. M.Sc. (Agri.) Thesis. University of
Agricultural Science, Dharwad.

Pawar, A. M. (2013). Knowledge and use of eco-friendly management practices by the mango growers of Konkan region. Ph.D. (Agri.) Thesis. Dr. Balasaheb Sawant Konkan Krishi Vidyapeeth, Dapoli.

Singh, K. V., Singh, G. P. and Priyadarshi, A. (2010). Extent of adoption of improved practices of mango production by mango growers in Muzaffarnagar district of Uttar Pradesh, India. Gujarat Journal of Extension Education. 18: 110-113.

Thorat, K. S. (2003). A study on technological GAP and constraints in adoption of recommended cultivation practices of mango growers. M.Sc. (Agri.) Thesis. Dr. Balasaheb Sawant Konkan Krishi Vidyapeeth, Dapoli.

\section{How to cite this article:}

Waghmod, Y. J., D. P. Hardikar and Haldavanekar, P. C. 2020. Adoption of Good Agriculture Practices by Mango Growers. Int.J.Curr.Microbiol.App.Sci. 9(04): 186-203.

doi: https://doi.org/10.20546/ijcmas.2020.904.024 